\title{
Dehydroepiandrosterone Sulfate Reduces Acoustic Injury of the Guinea-Pig Cochlea
}

\author{
Keiji Tabuchi ${ }^{1, *}$, Hidekazu Murashita ${ }^{1}$, Tadamichi Tobita ${ }^{1}$, Keiko Oikawa $^{1}$, Shigeki Tsuji ${ }^{1}$, \\ Isao Uemaetomari ${ }^{1}$, and Akira Hara ${ }^{1}$ \\ ${ }^{1}$ Department of Otolaryngology, Graduate School of Comprehensive Human Sciences, University of Tsukuba, \\ 1-1-1 Tennodai, Tsukuba 305-8575, Japan
}

Received May 2, 2005; Accepted August 19, 2005

\begin{abstract}
The present study was performed to determine effects of dehydroepiandrosterone sulfate (DHEAS), a neurosteroid, on acoustic injury. Albino guinea pigs were exposed to a $2 \mathrm{kHz}$ pure tone of 120 or $125 \mathrm{~dB}$ sound pressure level for 10 min immediately after intravenous administration of DHEAS. Statistically significant improvement in the compound action potential threshold shifts and in amplitude reduction of distortion-product otoacoustic emissions was observed 1 week after the acoustic overexposure in the animals treated with DHEAS. The present results suggest that DHEAS has a protective effect against acoustic injury of the cochlea.
\end{abstract}

Keywords: dehydroepiandrosterone sulfate (DHEAS), acoustic injury, cochlea

Dehydroepiandrosterone (DHEA, 3s-hydroxy-5androsten-17-one) and its sulfate ester dehydroepiandrosterone sulfate (DHEAS) are steroids secreted by the adrenal cortex in response to adrenocorticotrophine (ACTH). Evidence accumulated over the last decade has documented that the brain is capable of de novo synthesis of DHEA or DHEAS independent of adrenals (named neurosteroids). DHEA and DHEAS have recently received increased attention because it has been demonstrated that they display antioxidant properties and possess modulatory effects on NMDA, GABA, and sigma receptors and calcium channels (1). Thus, they have protective effects in various brain injuries, including glutamate excitotoxicity and anoxiareoxygenation $(2-4)$.

Evidence from previous studies suggests that oxidative stress and glutamate excitotoxicity are linked to the etiology of various cochlear injuries including acoustic injury. The protective effects of free radical scavengers and NMDA-receptor antagonists on acoustic injury have been demonstrated by several researchers $(5,6)$. The purported antioxidative and neuroprotective properties of DHEAS strongly suggest that DHEAS may possess protective effects in various cochlear injuries. However, to our knowledge, the effects of DHEAS on acoustic

*Corresponding author. FAX: +81-298-53-3147

E-mail: ktabuchi@md.tsukuba.ac.jp injury of the cochlea have never been examined.

It has been demonstrated that damage to outer hair cells (OHCs) is the initial effect of acoustic overstimulation (7). Distortion-product otoacoustic emissions (DPOAEs) are supposed to be closely related to an active process in the organ of Corti and are a useful test of $\mathrm{OHC}$ function. In the present study, we investigated the effect of DHEAS on acoustic injury. DPOAEs as well as compound action potential (CAP) were monitored before and after acoustic overexposure.

Studies were performed on albino guinea pigs weighing $250-350 \mathrm{~g}$. The animals were anesthetized with intraperitoneal injection of pentobarbital sodium (28 mg $/ \mathrm{kg}$, Nembutal ${ }^{\circledR}$; Abott, North Chicago, IL, USA). A catheter was inserted in the external jugular vein for administration of DHEAS. Body temperature was maintained at $37 \pm 1^{\circ} \mathrm{C}$ using a heating pad. All experimental procedures used in this study were approved by the Animal Experiment Committee at the University of Tsukuba.

CAP was recorded at the bony edge of the round window by an $\mathrm{Ag} / \mathrm{AgCl}$ electrode (8). The reference electrode was placed on the neck muscles. In order to evoke CAP, tone bursts of pure tones from 2 to $16 \mathrm{kHz}$ (rise and fall times, $1 \mathrm{~ms}$; duration, $10 \mathrm{~ms}$ ) were delivered in an open field system. Responses were averaged, and the sound pressure level necessary to 
Table 1. Frequencies and levels of $\mathrm{f} 1$ and $\mathrm{f} 2$

\begin{tabular}{lccc}
\hline $\mathrm{GM}(\mathrm{Hz})$ & $\mathrm{f1}(\mathrm{Hz} / \mathrm{dB} \mathrm{SPL})$ & $\mathrm{f} 2(\mathrm{~Hz} / \mathrm{dB} \mathrm{SPL})$ & 2f1 - f2 (Hz) \\
\hline 4000 & $3650 / 60$ & $4375 / 50$ & 2925 \\
6000 & $5475 / 50$ & $6575 / 40$ & 4375 \\
8000 & $7300 / 50$ & $8800 / 40$ & 5800 \\
\hline
\end{tabular}

GM: geometric means.

evoke $10 \mu \mathrm{V}$ CAP was defined as the threshold.

Because our recording methods for DPOAEs have been described elsewhere (9), only a brief summary will be given here. Two primary tones were delivered to the emitters contained within a custom-made ear probe. The frequencies and levels of the primary tones are shown in Table 1. DPOAEs were recorded by a microphone (EK-3024; Knowles, Itasca, IL, USA) inside the ear probe and processed by a spectrum analyzer (CF-350; Ono Sokki, Tokyo).

In order to induce acoustic trauma, the left ear was exposed to $2 \mathrm{kHz}$ pure tone of 120 or $125 \mathrm{~dB}$ SPL (sound pressure level) through the hollow ear bar for $10 \mathrm{~min}$. DHEAS was purchased from Sigma-Aldrich Japan (Tokyo). The animals were divided into the following 4 groups:

1) DHEAS-given $125 \mathrm{~dB}$ group $(n=12)$ : Each animal in this group was intravenously given $0.1(\mathrm{n}=5)$ or 1 $(\mathrm{n}=7) \mathrm{mg} / \mathrm{kg}$ DHEAS just before the acoustic overexposure of $125 \mathrm{~dB}$ SPL.

2) Physiological saline solution (PSS)-given $125 \mathrm{~dB}$ group $(n=7)$ : Each animal in this group received PSS intravenously just before the acoustic overexposure of $125 \mathrm{~dB}$ SPL.

3) DHEAS-given $120 \mathrm{~dB}$ group $(\mathrm{n}=12)$ : Each animal in this group was intravenously given $0.1 \mathrm{mg} / \mathrm{kg}(\mathrm{n}=5)$ or $1 \mathrm{mg} / \mathrm{kg}(\mathrm{n}=7)$ DHEAS just before the acoustic overexposure of $120 \mathrm{~dB}$ SPL.

4) PSS-given $120 \mathrm{~dB}$ group $(\mathrm{n}=7)$ : Each animal in this group received PSS intravenously just before the acoustic overexposure of $120 \mathrm{~dB}$ SPL.

All data were expressed as the mean \pm S.E.M. Shifts in the CAP thresholds and DPOAE amplitudes from the pre-exposure values were compared between the DHEAS- and the PSS-given groups using two-way ANOVA followed by the Scheffe test. The cochlear functions were evaluated at two time points after acoustic overexposure, specifically immediately after the acoustic overexposure to confirm the equality of acoustic overexposure between the groups and 1 week after the acoustic overexposure to evaluate the ultimate cochlear function (permanent threshold shifts after acoustic overexposure). A $P$ value of less than 0.05 was considered statistically significant.
Intravenous administration of $1 \mathrm{mg} / \mathrm{kg}$ DHEAS (a maximum dose dissolved in PSS) or $0.1 \mathrm{mg} / \mathrm{kg}$ DHEAS itself did not significantly affect the CAP threshold or DPOAE amplitude 10 min or 1 week after the administration ( $\mathrm{n}=4$ in each group, data not shown). The CAP threshold and amplitude of DPOAE were also not affected by $0.1 \mathrm{mg} / \mathrm{kg}$ DHEAS $(\mathrm{n}=3$, two-way ANOVA, data not shown).

Figures 1 and 2 show the CAP threshold shifts and reduction in DPOAE amplitude after the acoustic overexposure of $125 \mathrm{~dB}$ SPL, respectively. As shown in Figs. 1A and 2A, DHEAS did not affect shifts in the CAP threshold or DPOAE amplitude immediately after the acoustic overexposure. However, $1 \mathrm{mg} / \mathrm{kg}$ DHEAS $(\mathrm{n}=7)$ significantly decreased the CAP threshold shifts (Fig. 1B, two-way ANOVA: $P<0.01$, Scheffe test: $P<0.01$ in the $1 \mathrm{mg} / \mathrm{kg}$ subgroup) and significantly increased DPOAE amplitude 1 week after the acoustic overexposure (Fig. 2B, two-way ANOVA: $P<0.01$, Scheffe test: $P<0.01$ in the $1 \mathrm{mg} / \mathrm{kg}$ subgroup), although $0.1 \mathrm{mg} / \mathrm{kg}$ DHEAS $(\mathrm{n}=5)$ exhibited no such protective effects.

The results obtained in the $120 \mathrm{~dB}$ SPL experiment were essentially the same as those in $125 \mathrm{~dB}$ SPL. Namely, DHEAS significantly ameliorated the cochlear dysfunction at $1 \mathrm{mg} / \mathrm{kg} 1$ week after the acoustic overexposure, although $0.1 \mathrm{mg} / \mathrm{kg}$ DHEAS $(\mathrm{n}=5)$ did not affect the CAP threshold shifts or DPOAE amplitude (two-way ANOVA and Scheffe test: $P<0.01$ in the $1 \mathrm{mg} / \mathrm{kg}$ subgroup).

Several steroids, including DHEA, pregnenolone, progesterone, and their sulfate esters, can be synthesized within the central nervous system and are named neurosteroids. DHEAS has neurotrophic effects $(10,11)$ and modulatory functions on NMDA, GABA, and sigma receptors (1). Although DHEAS is a widely studied neurosteroid with multifunctional properties on the brain, effects of DHEAS on the cochlear function have not been fully determined. The present study showed that pretreatment with $1 \mathrm{mg} / \mathrm{kg}$ DHEAS significantly reduced the CAP threshold shift 1 week after acoustic overexposure. This finding is consistent with the observation on the cytoprotective effect of DHEAS in the central nervous system (12). To our knowledge, this study demonstrated for the first time that DHEAS exerted protective effects against acoustic injury of the cochlea. In addition, based on the results obtained in the DPOAE testing, DHEAS protected OHCs to alleviate CAP threshold shifts after acoustic injury.

It has been demonstrated that antioxidants and NMDA-receptor antagonists ameliorate acoustic injury of the cochlea $(5,6)$. Although relatively early production of free radicals in the cochlea after acoustic over- 

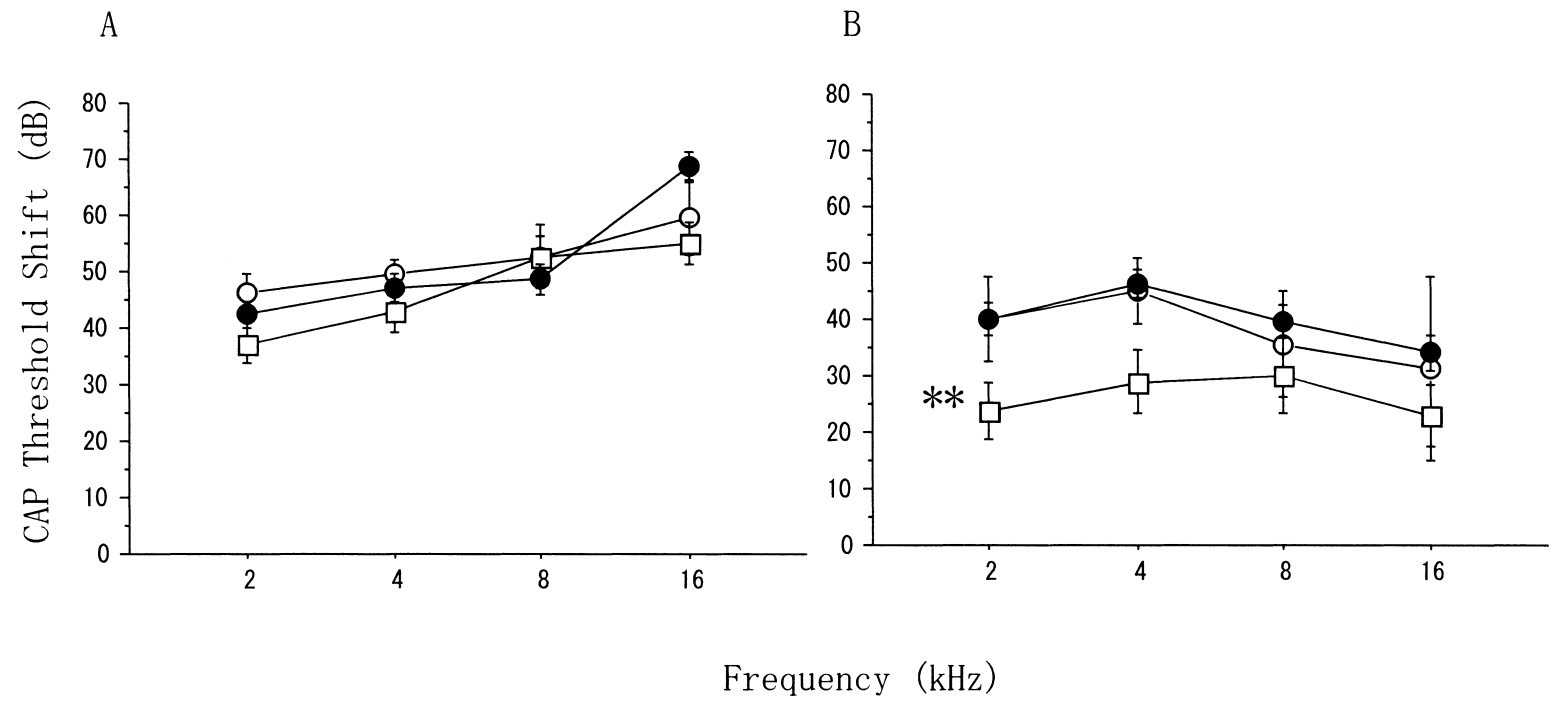

Fig. 1. Threshold shifts in CAP immediately (A) and 1 week (B) after the acoustic overexposure of $125 \mathrm{~dB}$ SPL. Closed circles indicate the PSS group, and squares and open circles indicate 1 and $0.1 \mathrm{mg} / \mathrm{kg}$ DHEAS groups, respectively. ${ }^{* *} P<0.01$, as compared with the PSS-group.
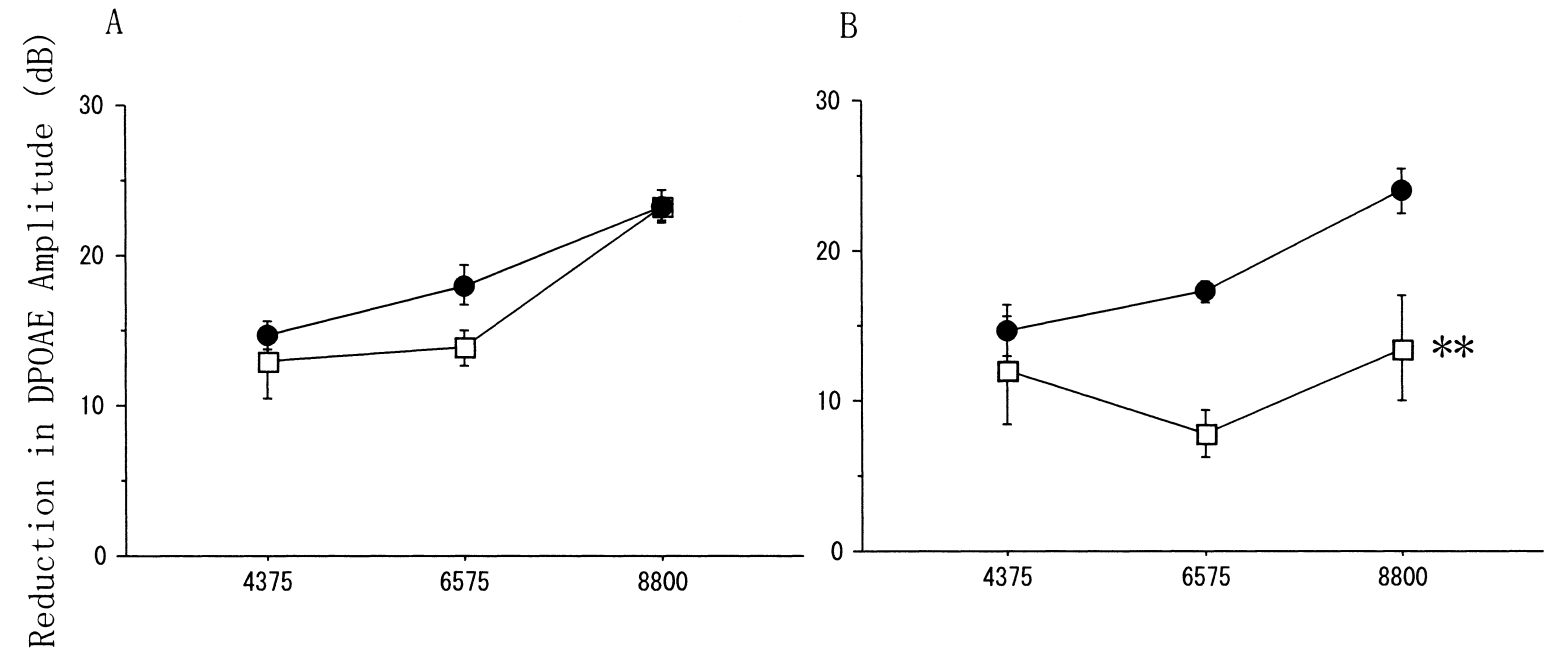

Frequency of $\mathrm{f} 2(\mathrm{~Hz})$

Fig. 2. Amplitude reduction in DPOAEs (dB re pre-exposure level) immediately (A) and 1 week (B) after the acoustic exposure of $125 \mathrm{~dB}$ SPL. Closed circles and open squares indicate the PSS group and $1 \mathrm{mg} / \mathrm{kg}$ DHEAS groups, respectively. ${ }^{* *} P<0.01$, as compared with the PSS-group.

exposure was demonstrated by Ohlemiller et al. (13), the precise time courses of free radical production and NMDA-receptor activation during and after acoustic overexposure have not been made clear. Published data have demonstrated that DHEAS acts to limit lipid peroxidation caused by free radicals $(12,14)$ and prevents glutamate and NMDA excitotoxicity (2). A receptor for DHEAS has not been definitively isolated yet (15), and up to now, these effects of DHEAS are considered to be non-genomic effects. Because nongenomic effects of steroids are generally observed within a short period, further studies will be necessary to elucidate the timing of free radical production and NMDA-receptor activation and to illustrate the precise protective mechanism(s) of DHEAS in acoustic injury.

In conclusion, besides the neuroprotective effects of DHEAS, we have shown here that DHEAS is able to protect the cochlea against acoustic injury. Functional 
findings have revealed that DHEAS protects OHCs to alleviate acoustic injury.

\section{Acknowledgments}

This work was supported by a Grant-in-Aid for Scientific Research for Young Scientists (B)16790984 from the Ministry of Education, Culture, Sports, Science, and Technology of Japan. The authors are grateful to Mr. Kwang K. Pak (University of California, San Diego) for his help on this paper.

\section{References}

1 Baulieu EE, Robel P. Dehydroepiandrosterone and dehydroepiandrosterone sulfate as neuroactive neurosteroids. J Endocrinol. 1996;150:S221-S239.

2 Kimonides VG, Khatibi NH, Svendsen CN, Sofroniew MV, Herbert J. Dehydroepiandrosterone (DHEA) and DHEA-sulfate (DHEAS) protect hippocampal neurons against excitatory amino acid-induced neurotoxicity. Proc Natl Acad Sci USA. 1998;95:1852-1857.

3 Aragno M, Parola S, Brignardello E, Mauro A, Tamagno E, Manti R, et al. Dehydroepiandrosterone prevents oxidative injury induced by transient ischemia/reperfusion in the brain of diabetic rats. Diabetes. 2000;49:1924-1931.

4 Morin C, Zini R, Simon N, Tillement JP. Dehydroepiandrosterone and alpha-estradiol limit the functional alterations of rat brain mitochondria submitted to different experimental stresses. Neuroscience. 2002;115:415-424.

5 Duan M, Agerma, K, Ernfors P, Canlon B. Complementary roles of neurotrophin 3 and a N-methyl-D-aspartate antagonist in the protection of noise and aminoglycoside-induced ototoxicity.
Proc Natl Acad Sci U S A. 2000;97:7597-7602.

6 Ohinata Y, Miller JM, Schacht J. Protection from noise-induced lipid peroxidation and hair cell loss in the cochlea. Brain Res. 2003;966:265-273.

7 Boettcher FA, Spongr VP, Salvi RJ. Physiological and histological changes associated with the reduction in threshold shift during interrupted noise exposure. Hear Res. 1992;62:217-236.

8 Tabuchi K, Tsuji S, Fujihira K, Oikawa K, Hara A, Kusakari J. Outer hair cells functionally and structurally deteriorate during reperfusion. Hear Res. 2002;173:153-163.

9 Tabuchi K, Okubo H, Fujihira K, Tsuji S, Hara A, Kusakari J. Protection of outer hair cells from reperfusion injury by an iron chelator and nitric oxide synthase inhibitor. Neurosci Lett. 2001;307:29-32.

10 Compagnone NA, Mellon SH. Dehydroepiandrosterone: a potential signaling molecule for neocortical organization during development. Proc Natl Acad Sci U S A. 1998;95:4678-4683.

11 Roberts E, Bologa L, Flood JF, Smith GE. Effects of dehydroepiandrosterone and its sulfate on brain tissue in culture and on memory in mice. Brain Res. 1987;406:357-362.

12 Bastianetto S, Ramassamy C, Poirier J, Quirion R. Dehydroepiandrosterone (DHEA) protects hippocampal cells from oxidative stress-induced damage. Brain Res Mol Brain Res. 1999;66:35-41.

13 Ohlemiller KK, Wight JS, Dugan LL. Early elevation of cochlear reactive oxygen species following noise exposure. Audiol Neurootol. 1999;4:229-236.

14 Boccuzzi G, Aragno M, Seccia M, Brignardello E, Tamagno E, Albano E, et al. Protective effect of dehydroepiandrosterone against copper-induced lipid peroxidation in the rat. Free Radic Biol Med. 1997;22:1289-1294.

15 Widstrom RL, Dillon JS. Is there a receptor for dehydroepiandrosterone or dehydroepiandrosterone sulfate? Semin Reprod Med. 2004;22:289-298. 